\title{
HÉRITER, LUTTER, ÉPROUVER: DES NOTIONS POUR INTERROGER LA SANTÉ COMME TRACE. ${ }^{1}$
}

\author{
Isabelle Gobatto \\ isabelle.gobatto@u-bordeaux.fr \\ Doutora em sociologia pela Université de Paris V, Professora da Université de \\ Bordeaux (França) \\ ORCID: https://orcid.org/0000-0002-6570-6173
}

Je souhaite tout d'abord remercier mes collègues de m’avoir invité à prendre part à ce congrès pour en prononcer la conférence de clôture. C’est un très grand honneur et un plaisir pour moi de venir à la rencontre de mes collègues brésiliens, d'autant que je mesure vraiment la différence de culture disciplinaire entre nous. Vous proposez une anthropologie qui me semble inclure très naturellement la question de l'engagement du chercheur, presque comme une évidence, mais c'est une évidence construite dans des ancrages sociaux et historiques. L'anthropologie française me semble bien plus timide dans ce registre. Cela produit des sujets d'étude et des manières de les interroger vraiment différents.

Alors justement, pour mettre en dialogue ces deux contextes de la pratique anthropologique dans le domaine de la santé, je propose d'articuler cette communication autour de trois notions particulièrement présentes et très heuristiques dans le champ contemporain de l'anthropologie de la santé, notamment en France: hériter, lutter, éprouver. Ce sont des notions qui traversent mes propres interrogations d'anthropologue de la santé et d'africaniste. Pour résumer rapidement ici, à travers la santé, j'interroge plus fondamentalement les rapports des individus au monde et à 'leur monde', puisque le monde n'est pas une donnée de la nature mais une construction. Je mattache plus précisément à étudier celui que nous construisons par le biais de la santé. Et dans ce vaste domaine, jétudie plus particulièrement l'exercice contemporain de la biomédecine, lélaboration des savoirs engagés dans le travail médical quotidien, en particulier, depuis quelques années, face aux maladies chroniques non transmissibles qui sont en expansion dans le monde et dans les sociétés ouest-africaines où je travaille. Un des caractères communs de ces sociétés est dêtre marquées par la pauvreté structurelle. Je questionne l'entrelacement de ces deux phénomènes: pauvreté et travail médical. J'interroge aussi le rôle des différents savoirs dont nous pouvons disposer, dans la construction de ces mondes tels qu'ils sont pensés et tels qu'ils sont vécus. En outre, dans mon travail, le corps est en quelque sorte le noyau dur qui ramasse tous mes questionnements puisque c'est cette base matérielle qui nous permet déprouver le monde, d'y construire des expériences dont celles qui concernent la santé et la maladie, la souffrance... Le corps matérialise notre lien au monde en 
tant que réalité mais aussi en tant que projet. Il en est un vecteur et, du coup, il en est une trace, pour l'anthropologue.

Ces trois notions: hériter, lutter, éprouver, dont on peut changer l'ordre à léchelle d'une vie, sont intéressantes parce qu'on peut les mobiliser à différentes échelles justement, celle d'une vie, d'un individu. Elles engagent des problématiques d'histoire et de mémoire dans lesquelles on s'ancre pour construire le présent; elles renvoient aux différents types de luttes pour espérer rester en vie. Elles évoquent les affects, les émotions qui s'attachent au corps malade, au corps sous traitement. On peut mobiliser aussi ces notions à léchelle d'un groupe ou d'une société: elles renvoient alors aux problématiques de mobilisation, dactivisme pour atteindre ce à quoi on croit, ce que l'on souhaite pour soi, pour son ou ses groupes d'appartenances. Il me semble que tout ce qui concerne les droits sexuels, les discriminations à partir d’orientations sexuelles, la question des violences associées à certaines pratiques thérapeutiques, le racisme - des thématiques qui ont donné lieu à des échanges dans ce congrès -, engagent de manière complexe ces notions à l'interface de l'individuel et du collectif. Et elles sont intéressantes aussi à l'échelle de leur pertinence dans les sociétés dites du sud, prises dans ce mouvement de santé globale, en partie régulée par les organismes internationaux et à l'échelle desquels nos héritages culturels, nos luttes sociales, nos expériences vécues sont aussi en question, notamment pour penser le rôle que des spécificités sociales et culturelles peuvent jouer dans ce monde global s'agissant des questions de santé. La problématique des savoirs indigènes est par exemple intéressante à considérer à cette échelle de production de légitimité oud 'invisibilité. Ces trois notions sentrelacent et font donc trace pour l'anthropologue lorsqu'il tente de rendre compte de ces mondes sociaux. C’est ce que je vais essayer de développer dans cette communication de clôture.

Un rappel: la santé et toutes ses ramifications qui engagent le corps et plus précisément les expériences corporelles en matière de sexualité, de fabrique des identités et du genre, de production des savoirs, des normes, du droit qui institue des pratiques légitimes etc., renvoient à des expériences intimes, attachées à des pratiques individuelles et collectives, à des institutions qui encadrent ces expériences à étudier finement à partir d'un ensemble d'interactions soutenues entre le chercheur et ses interlocuteurs. Mais il s'agit aussi d'objets scientifiques, donc de constructions à partir desquelles le chercheur étudie les mondes dans lesquels nous vivons, les sociétés dans leur organisation sociale et culturelle, dans les modalités de leur pérennité mais aussi dans leurs temps de ruptures, de décrochages. Le domaine de la santé a ceci d’extraordinaire qu'il ouvre sur des perspectives excessivement porteuses précisément pour 'dire' ces mondes dans lesquels nous vivons mais aussi dans lesquels nous souhaitons vivre. Tous les échanges qui ont concerné les droits sexuels, les problématiques de discrimination, de violence associées aux pratiques 
qui engagent le corps, de légimitation de savoirs indigènes durant ce congrès sont particulièrement illustratifs de cette posture épistémologique et éthique de l'anthropologue qui certes 'rend compte', participe à 'faire comprendre', mais qui, dans un même mouvement, prend une place dans ce processus. Ceci, qu'il le revendique, ou pas.

\section{HERITER}

Depuis les années 1990, je conduis des recherches sur l'exercice contemporain de la biomédecine à partir de terrains multi-situés en Afrique de l'Ouest francophone. La biomédecine et le travail des professionnels de santé en Afrique est un objet qui est longtemps resté impensé dans l'anthropologie française, au profit de létude des tradimédecines et de leurs savoirs. L'intérêt des anthropologues français a été tardif pour la médecine et les institutions de santé comme l'hôpital en Afrique. Il faut dire que les théories d'inspirations fonctionnalistes ont longtemps occupé le devant de la scène, soulignant des systèmes normatifs d’attentes auxquelles des fonctions professionnelles venaient soumettre les soignants, où qu'ils exercent, mais ce ne sont pas des théories qui contextualisent. Or, en Afrique comme au Brésil, ou dans le monde occidental, l'exercice de la médecine repose sur des savoirs, des pratiques thérapeutiques, des relations de soin qui sont pénétrés par l'histoire, par les cultures, par le politique et bien sûr par des dimensions économiques documentées qui agissent à différentes échelles, des plus globales au plus locales.

Dans cet ensemble, la question de l'héritage est au cœur de l'exercice de la biomédecine où qu'elle s'exerce, et donc en corollaire au cœur des faits de santé. Mais comment? Dans nombre de pays, y compris en Afrique de l'Ouest francophone, l'héritage qui participe à fonder les pratiques de soin et ses institutions, dont le droit qui est au cœur de ce congrès, prend racine ailleurs. Les savoirs de la biomédecine reposent sur les sciences biologiques, sur une manière précise de 'faire la preuve', qui s'est structurée ailleurs et qui repose avant tout sur des essais cliniques conduits sur de très grands échantillons. D’ailleurs, ce processus s'accentue avec ce qu'on nomme l'Intelligence Artificielle, en pleine expansion dans le domaine de la santé. Elle s’appuie sur des algorithmes, eux-mêmes ancrés dans ce qu’on appelle communément les big data, puisque l'I.A. brasse des milliers d'informations extraites de dossiers médicaux de patients. Cela permet la production de savoirs qui évacuent en quelque sorte l'humain pour se fixer sur le corps, pour calculer des risques de survenue de maladies, la valeur de certains traitements, des effets secondaires de médicaments, mais ceci en produisant des modèles: l'Intelligence Artificielle est aujourd'hui au centre de la recherche médicale en France. Ce sont ces formes de production de savoirs qui font de plus en plus autorité dans la biomédecine et ses institutions. A tel 
point que le gouvernement français a placé la santé en tête des secteurs prioritaires pour le développement de l'Intelligence Artificielle.

Parallèlement, la tendance à évacuer les données culturelles des savoirs et du raisonnement de soin, s'amplifie. Dans ce modèle biomédical, ce sont les médecins qui ont lautorité sur la définition de la maladie et sur son traitement. Suivant des dynamiques historiques et politiques que je ne développerai pas ici, les praticiens ont été institués en professionnels légitimes pour définir les critères du normal et du pathologique, pour distinguer l'état de santé des états de maladie et pour octroyer à une personne un statut de malade lui ouvrant ensuite des droits sociaux. Tout ce processus a une histoire essentiellement occidentale qui diffuse au cœur du modèle biomédical. Et nous en héritons, mais pas sous les mêmes modalités selon les contextes culturels dans lesquels nous évoluons. Pour le dire rapidement, dans les sociétés occidentales, nous sommes socialisés dès notre enfance avec ce modèle, il s'inscrit dans nos dispositions à penser le corps et les savoirs liés à la santé. Nous héritons de cette histoire sociale qui se pérennise ensuite parce qu'elle est inscrite dans une variété d'institutions qui s'y ajustent, de dispositifs, de rituels etc., dont l'une des fonctions est précisément de maintenir ces savoirs à leur place, de pérenniser ce fonctionnement. Me concernant, je continue à documenter comment la profession médicale, en Afrique francophone, s'invente par rapport à cet héritage bien plus récent à l'échelle de l'histoire des sociétés, sur ce continent. Face à cette situation en quelque sorte d'hégémonie de certains savoirs qui nont plus à faire leur preuve, la notion d'héritage est intéressante parce quelle permet de relire la question de la reconnaissance différenciée des savoirs, en particulier des savoirs locaux ou indigènes en matière de soin du corps et en matière de santé dont il a été question dans ce congrès et celle de la diversité thérapeutique également discutée ici. On peut considérer sous ce prisme certaines des multiples conséquences de cette histoire héritée parmi lesquelles un déclassement des savoirs populaires sur le corps et les maladies, élaborés et formulés par différentes communautés, mais également celui de leurs modèles explicatifs de la maladie. Car classement et déclassement, mise à l'écart et relégation vont ensemble. Cet héritage, effectif aujourd'hui bien que différencié selon les contextes, laisse des traces portées individuellement et collectivement parmi lesquelles il existe une difficulté à ce que se déploie une mixité des savoirs dans certaines institutions de santé; ceci, par contre, de manière terriblement commune et transversale à nombre de sociétés très différenciées par ailleurs sur les plans culturel et historique. Où en est-on aujourd'hui de ces situations? Comment la mise en dialogue de situations sociales différentes, comme lors de ce congrès, pourrait éclairer ce phénomène transversal à nombre de sociétés, en souligner certains agencements et, dans une dimension plus appliquée, contribuer à les réajuster? Il me parait intéressant de se donner pour objet de mettre à jour toutes les traductions 
physiques et spatiales de cette situation de reflux, de mise à distance de certaines formes de savoir. Il faut donc se pencher sur les multiples manières dont ce binôme classement-déclassement devient pratique, comment il se cristallise dans des corps, se déploie dans des lieux, dans des pratiques sociales y compris lorsqu'elles s'annoncent comme les plus favorables à ces autres formes de savoir sur le corps et la santé. Comme par exemple à travers leur confinement, sous prétexte de valorisation ou de préservation, dans des lieux ou dans des communautés: des communautés indigènes avec des savoirs locaux, des services de médecine interculturelle où l'on sait écouter les cultures des patients. On pourrait aussi se demander comment une anthropologie qui sollicite les dimensions politiques des rapports nord-sud pourrait venir renforcer ce projet de dévoilement.

Il en va de même de la notion du corps légitime, autre thème de ce congrès. Comme l'ont étudié nombre de sociologues, rien n'est plus classant que l'hexis corporelle, la façon dont le corps est tenu, vêtu. On le sait depuis longtemps, les écarts sociaux se traduisent dans des écarts aux modèles corporels légitimes. Et comme pour les savoirs, il y a des corps dominants, dans leur phénotype, dans les représentations qui s'y attachent, les traitements auxquels ils sont culturellement soumis, produits d'une construction historique qui s'impose avec autorité à d'autres pour instaurer le contenu social de ces corps légitime et, en corollaire, des manières dont ils doivent être appréhendés. Ce qui est très troublant pour moi, c'est d'étudier ce classement-déclassement qui devient d'autant plus efficace dès lors qu'il est inscrit dans les routines mentales de ceux-là mêmes qui ont vu leurs savoirs déclassés. Pour exemple, les médecins africains avec lesquels je conduis des recherches sont souvent particulièrement virulents sur "ces africains qui ne comprennent rien", "ne savent pas prendre soin de leur corps", leurs propres patients... Euxmêmes étant des africains! Mais il y a cette nécessité de s’identifier à une communauté scientifique internationale dont ils ont acquis une part d'identité par leur profession. Il y a là, dans ces impensés, une illustration des rouages centraux de cet héritage dans ses efficiences contemporaines telles qu'elles se déploient dans nombres de sociétés des suds.

Donc la notion d'hériter est intéressante parce quelle parle de notre passé inscrit dans notre présent, notamment via les savoirs. Mais elle parle aussi de notre avenir en tant quêtres sociaux: comment construiton nos héritiers? Ceux qui demain, vont poursuivre notre travail? Que leur transmettons-nous? Comment marquons-nous l'esprit des générations en devenir face aux questions de santé? Là aussi la santé rencontre les problématiques déducation. Je soutiens qu'il y a deux grands domaines et leurs institutions qui incarnent tout particulièrement une culture et son devenir en lien avec cette question de l'héritage: la façon dont nous traitons les corps affectés et la façon dont nous structurons léducation de nos enfants par lesquels nous inscrivons - nous maté- 
rialisons - le projet d'une société, y compris s'agissant des futurs corps légitimes. Peut-être y a-t-il place pour de nouvelles représentations de nous-mêmes? C'est ce vers quoi nous mènent les combats LGBT, parmi d'autres luttes. Alors lutter, précisément.

\section{LUTTER}

Je le disais tout à l'heure, les temps de décrochage, les changements dans une société sont souvent articulés à des formes de luttes, instituées ou pas, dont l'activisme et le militantisme sont des modalités discutées aussi dans ce congrès. Le sida l’a particulièrement illustré dans le domaine de la santé, montrant des sociétés qui glissent de la gestion de corps marqués, déclassés, à des corps que certaines luttes collectives ont enfin rendus visibles: celles-ci et d'autres témoignent des fonctions de ces luttes en tant que des temps forts d'expression des projets que vise une société. C’est donc un domaine deétude important, central pour les anthropologues. Qu'est-ce qui est construit en problème? En intolérable? A l'échelle individuelle, à léchelle collective? Qu’est-ce qui crée de la mobilisation, qu'est-ce qui n’en crée pas et qu'est-ce que ça nous apprend?

Par exemple, actuellement, le champ des Disability Studies émerge tout juste en Europe, en confrontant les politiques publiques dans ce domaine aux droits fondamentaux internationaux dans la filiation de ce qui s'est produit pour le sida. Beaucoup de travaux se développent autour des problématiques d’autonomie. Le traitement social du handicap montre comment une société traite l'étrangeté et crée de l'invisibilité. Les personnes handicapées mentales face au risque de contracter le VIH, par exemple, ou les personnes âgées, absentes des discours de prévention comme si la société française était incapable de penser que ces personnes ont une sexualité. Et une valeur qui mérite qu’on continue à envisager la prévention pour elles. Ou par exemple, encore en France, Alzheimer, c'est 20000 patients qui ont moins de 60 ans. La plus jeune a moins de 30 ans! Cela n'a fait naitre, jusquà ce jour, aucune action collective pour accompagner les aidants pourtant très sollicités, avec un rôle central dans les prises en charge.

Proposer de retenir cette notion, lutter, c'est aussi rappeler à quel point lutter est une attitude, une prise de position et une condition, vraisemblablement ressenties, exprimées, organisées par une bonne partie de l'humanité, même si cela ne se fait pas nécessairement de la même façon partout. Il y a là, de nouveau, une forme de continuité très intéressante à étudier, dans ses spécificités selon les contextes socioculturels et dans ses points de rencontre entre sociétés. Nos collègues paléoanthropologues souligneraient qu'on peut faire remonter l'action de lutter à nos ancêtres Homo Sapiens. Cela leur a permis de se maintenir et de se développer pendant des millénaires. Et la lutte, y compris d’ailleurs pour 
la nature, pour l'environnement, est aussi un élément marquant du $21^{\text {ème }}$ siècle. Alors pour quoi, pour qui lutte-t-on aujourd'hui concernant les questions de santé et de droit? Comment? Et de nouveau quest-ce que les mises en dialogues de situations ici au Brésil, en France, aux EtatsUnis, peuvent nous apprendre?

Comme pour les questions d'héritage, travailler, en anthropologie, à saisir les objets des luttes individuelles et collectives dans une société donnée et entre sociétés, c’est se donner les moyens de faire affleurer le permanent sous le contingent, l'universel sous l'individuel. En même temps, c'est une voix intéressante qui soumet à la discussion les traitements sociaux différenciés de cette permanence. En matière de précarité des existences et de pauvreté, par exemple, en matière de souffrances que les situations de précarité infligent aux hommes lorsqu'ils ne peuvent pas ou plus se faire soigner alors que les soins pour leur problème de santé existent et sont disponibles pour d'autres qu'eux-mêmes. Cette précarité des vies est une donnée centrale dans les sociétés d’Afrique subsaharienne, c'est en y travaillant que j’ai ressenti la nécessité de documenter comment les dynamiques de la pauvreté s'infiltraient dans le travail médical. Les situations de pauvreté non seulement réduisent les possibilités de délivrer des soins pour les soignants, mais aussi, pour les malades, de recourir aux soins. Tout cela est bien documenté. Mais ces situations pénètrent aussi les façons de penser la pratique médicale chez les médecins et les infirmiers: en particulier les notions de responsabilité envers les malades, de respect, de déontologie la notion d’erreur médicale aussi, qui ne devrait pas engager la responsabilité du soignant puisqu'il na pas les moyens de bien faire son travail! D’ailleurs cette notion n'est pas présente dans l'enseignement dans les pays où j’ai travaillé. Dans un autre registre, dans le cadre de la prise en charge du diabète de type 2 , javais noté que les médecins, au Mali, étaient tellement critiques sur leurs patients parce qu'ils ne venaient pas régulièrement faire vérifier leur glycémie, qu'ils étaient incapables de "les voir" lutter pour éviter telle complication, pour payer l'insuline, pour manger mieux; lutter pour faire ce qu'on attend d'eux en tant que patients. De nouveau, leurs luttes étaient invisibles. Je pense notamment à la charge émotionnelle, aux mobilisations au sein des familles en situation de précarité afin de faire soigner un enfant atteint d'une maladie chronique, obtenir l'insuline, faire des examens pour orienter une prise en charge, etc. Quand on lit, par ailleurs, des ouvrages comme ceux Julie Livingston, Improvising medicine ${ }^{2}$, une ethnographie dans un service d'oncologie au Botswana, la chercheuse montre une médecine du tri, ce qu’a aussi étudié Vinh Kim Nguyen face au sida ${ }^{3}$. Les médecins trient entre les malades quon ne peut plus prendre en charge parce qu'ils ne peuvent plus payer ou parce que la maladie continue à évoluer malgré les traitements et on les renvoie chez eux mourir pour libérer des lits d'hôpitaux. Julie Livingston étudie tous ces sentiments mêlés, l'espoir, la souffrance, la révolte, la résignation chez les professionnels comme chez les malades. Le triage, je le rappelle, c'est ce qui se fait en temps de guerre, 
lorsqu'il y a un afflux massif de blessés que les chirurgiens opèrent à la chaine; il faut classer 'en attente' les soldats les plus gravement atteints, ce qui revient à les laisser mourir. C'est un terme français qui a fait son apparition dans les traités de médecine militaire au $19^{\text {ème }}$ siècle. Le contexte du combat et son urgence rendent sans doute ces décisions nécessaires, mais le contexte de pauvreté? Il faut croire que nombre de pays d'Afrique sont en guerre parce qu'ils pratiquent le triage sans lui donner ce nom. Notamment pour les cancers, qui nécessitent des soins coûteux et longs. Les cancers, entre parenthèse, sont en train de redessiner les contours de la santé globale, dans les pays du sud. Lanthropologue, de nouveau, constate la valeur différentielle des vies, bien qu'il n’ait pas à juger. Mais qui lutte pour faire changer cela? Où sont les luttes?

Jaimerais vraiment comprendre finement ce que les situations de pauvreté construisent et produisent, de manière de nouveau transversale aux sociétés et qui passe par le domaine des savoirs, valeurs, des situations classées comme intolérables ou acceptables. Je suis certaine que des mises en perspective avec des situations ici, au Brésil, pourraient permettre de faire émerger de nouvelles hypothèses de travail. Pour le cure et pour le care, le prendre soin. Lanthropologie du care est importante au Canada, aux Etats-Unis, un peu plus récente en France, et quasiment absente des recherches menées par les anthropologues africanistes. C'est quand-même intéressant à noter!!

Ces quelques exemples me permettent de poser une question épistémologique un peu provocatrice: cette lutte peut-elle - et doit-elle prendre une place dans le travail de l'anthropologue? Cela renvoie à la définition de la discipline, de ses contours légitimes et à la question de l'engagement du chercheur, savoir sur quelles scènes sa propre lutte peut s'exprimer. Il n'y a pas de consensus sur ces questions qui sont clairement articulées à des cultures disciplinaires. Parfois, le travail de certains anthropologues s'articule 'naturellement' à des formes d'activisme, à des mobilisations parce que dire le monde tel qu'il est, rendre compte, témoigner sur les scènes académiques et dans la cité, c'est tout cela ensemble qui les constituent en tant que personne. Parfois pas. Pour diverses raisons que, de nouveau, il n'est pas question de juger. Mais cela ouvre un débat sur la place que peut occuper l'anthropologue dans le changement socioculturel.

\section{EPROUVER}

Eprouver, c'est une manière de percevoir le monde, de le juger, de s'y comporter; cela passe par le corps, sans qu'il ait la nécessité de mots, en première intention. Sans la nécessité de l'explication. C'est ce par quoi un objet ou une situation se rendent présents à lesprit du chercheur, par le biais de lémotion qui nait et cette émotion va alerter, par exemple, sur des objets à considérer. Cela a été documenté: en tant quêtre sociaux, 
nous ressentons beaucoup de situations et au-delà nous les comprenons sans passer par le registre de l'intellect, dans un premier temps. C'est l'affect qui signifie. Et il signifie à partir d'un socle commun d'expériences ressenties, déjà là, pré-dimensionnées comme l’a développé notamment Françoise Héritier dans certains de ses travaux. Cette place des affects, de ce que l'on éprouve, fait qu'on n'a pas besoin de convoquer consciemment tous les liens, de les décortiquer pour saisir une situation, une histoire, un fait raconté. Françoise Hériter expliquait, dans l'un de ses derniers ouvrages, que ce sont les expressions toutes faites que l'on emploie, que toutes les personnes qui partagent un même environnement culturel comprennent, par exemple elle cite en français: "je te garde un chien de ma chienne" pour dire qu'on est rancunier et qu'on va se venger un jour ou l'autre. Ou "tu ne fais pas dans la dentelle" pour dire que quelqu'un est un peu grossier. Nous comprenons tous le double sens de ces expressions, dès lors que nous les 'connaissons' depuis notre enfance. Nous avons tous en nous, ce processus que F. Héritier appelle "des court-circuits de la pensée" qui font qu'on n'a pas besoin du recours à la pensée consciente pour comprendre quelque chose et pour ressentir. Eprouver, c'est la traduction intime de notre relation au monde, en fait. Le domaine de la santé en est pétri.

Ce qui est intéressant, c'est que les émotions sont uniques et, en même temps, elles sont aussi un aspect saillant de nos vies dêtres humains. Comme les deux premières notions évoquées. Les émotions sont un registre que nous partageons d'ailleurs avec d'autres espèces animales. Travailler sur les peurs, par exemple, ou la colère, le plaisir, le doute. Ce sont des objets qui nous permettent d'accéder au sens profond de ce qui est vécu: en se soignant, en rencontrant la maladie, le risque de mort, etc. Les émotions sont d'ailleurs plus importantes qu’on ne l'imagine dans la structuration des sociétés. Prenons les hôpitaux: à l'origine, ceétaient des organismes de bienfaisance qui ont été ensuite gérés par des professionnels afin de répondre au sentiment d'injustice face à la maladie. Emotion et sentiments, ce n'est pas tout à fait pareil: les sentiments se communiquent par le langage, ce sont les émotions mises en mots.

Chaque fois que je fais du terrain en Afrique, je ressens des bouffées de colère face à ce que jobserve parfois, que je vais nommer ensuite. Sentiment de colère, de peine, une fois lémotion éprouvée. Mais être 'affecté, ce n'est pas juste une épreuve pour le chercheur. Ce qui est plus important c'est que l'affect signale que quelque chose se passe. Les émotions et les sentiments qui les mettent en mots sont de plus en plus présents dans les réflexions des anthropologues français, qui ont tendance à considérer la question sous deux angles : dans le cadre de létude de leurs objets de travail, bien sûr. Pour ma part, je m’intéresse au registre de sensibilités des soignants. Je me demande en particulier, parce que je suis un peu monomaniaque, comment la pauvreté impacte la palette des émotions que peuvent ressentir les soignants dans leur travail. 
Qu'est-ce qui les émeut, ces soignants? Qu'est-ce qui les met en colère? Qu'est-ce qui leur procure de la joie dans leur travail? Second angle, on revient sur la place que les émotions occupent dans le déroulement du travail de l'anthropologue: nos propres affects peuvent parfois être une condition de la connaissance. Par exemple, lorsqu'on prend conscience de l'imminence d'une réaction dictée par notre corps en situation d'observation ou d'entretien, cette émotion va nous alerter sur des objets à considérer, des questions à poser. Je dois dire que jéprouve du plaisir à penser qu'intégrer nos émotions à notre questionnement scientifique, c'est s'autoriser à penser le travail de l'anthropologue autrement que par la nécessité d'une objectivation pleine et entière. Quand lémotion surgit, il n'est pas question de rationalité, d’objectivité et pourtant on peut s'en servir sans fragiliser lobjectivité du travail. Ce qui fait de la notion d'éprouver une notion aussi intéressante à étudier sur un plan théorique que sur un plan méthodologique. Aujourd'hui c'est devenu un objet de réflexion légitime pour la plupart des sciences sociales, dont l'anthropologie. Alors qu'est-ce qui nous émeut au $21^{\text {ème }}$ siècle en tant qu'anthropologues? Pour moi c'est une question essentielle à se poser et sur laquelle on échange insuffisamment. On produit de la recherche parce qu'on répond à des appels d’offre, parfois; on va parfois vers là où le financement nous porte. Mais qu'est-ce qui nous touche? Il y a là en tout cas, matière à faire se rencontrer l'anthropologie des émotions et l'anthropologie de la santé, notamment autour des questions de mouvements sociaux et de droit, thèmes qui étaient au cœur de ce congrès.

Je vais conclure en quelques mots. J'ai choisi de discuter des questions de santé en recourant à des ressources conceptuelles qui débordent très largement ce domaine parce qu'elles parlent plus fondamentalement de manières dêtre au monde dont la santé n'est qu'une expression parmi d'autres et qui mobilise des dynamiques que l'on retrouve ailleurs. Hériter, transmettre, se souvenir, inventer, éprouver, se mobiliser sont autant de manifestations qui se déclinent à l'infini à l'échelle de nos vies individuelles, mais c'est aussi ce qui nous définit intrinsèquement en tant quêtres humains. Et là l'objet deétude 'santé, d'une part, et l'anthropologie fondamentale d'autre part, s'enrichissent vraiment l'une par l'autre.

\section{NOTAS}

1. Este artigo é a versão reformulada da conferência de encerramento da III Reunião de Antropologia da Saúde organizada entre os dias 23 a 25 de setembro de 2019, na Universidade Federal do Rio Grande do Norte (Campus Natal), que teve como tema: "Saúde, Movimentos Sociais e Direitos". Texto revisado por Julie Cavignac.

2. LIVINGSTON, Julie. Improvising Medicine. Durham: Duke University Press, 2012.

3. NGUYEN, Vinh-Kim. The republic of therapy: triage and sovereignty in west Africa's time of AIDS. Durham: Duke University Press, 2010. 